\title{
Investigating the effect of two methane-mitigating diets on the rumen microbiome using massively parallel sequencing
}

\author{
E. M. Ross, ${ }^{\star} \dagger \ddagger^{1}$ P. J. Moate, $\S$ L. Marett, $\S$ B. G. Cocks, ${ }^{*} † \ddagger$ and B. J. Hayes ${ }^{*} \dagger \ddagger$ \\ *Biosciences Research Division, Department of Primary Industries, Bundoora, VIC 3083, Australia \\ †Dairy Futures Cooperative Research Centre, Bundoora, VIC 3083, Australia \\ łLa Trobe University, Bundoora, VIC 3086, Australia \\ §Future Farming Systems Division, Department of Primary Industries, Ellinbank, VIC 3820, Australia
}

\begin{abstract}
Variation in the composition of microorganisms in the rumen (the rumen microbiome) of dairy cattle (Bos taurus) is of great interest because of possible links to methane emission levels. Feed additives are one method being investigated to reduce enteric methane production by dairy cattle. Here we report the effect of 2 methanemitigating feed additives (grapemarc and a combination of lipids and tannin) on rumen microbiome profiles of Holstein dairy cattle. We used untargeted (shotgun) massively parallel sequencing of microbes present in rumen fluid to generate quantitative rumen microbiome profiles. We observed large effects of the feed additives on the rumen microbiome profiles using multiple approaches, including linear mixed modeling, hierarchical clustering, and metagenomic predictions. The effect on the fecal microbiome profiles was not detectable using hierarchical clustering, but was significant in the linear mixed model and when metagenomic predictions were used, suggesting a more subtle effect of the diets on the lower gastrointestinal microbiome. A differential representation analysis (analogous to differential expression in RNA sequencing) showed significant overlap in the contigs (which are genome fragments representing different microorganism species) that were differentially represented between experiments. These similarities suggest that, despite the different additives used, the 2 diets assessed in this investigation altered the microbiomes of the samples in similar ways. Contigs that were differentially represented in both experiments were tested for associations with methane production in an independent set of animals. These animals were not treated with a methane-mitigating diet, but did show substantial natural variation in methane emission levels. The contigs that were significantly differentially represented in response to both dietary additives showed
\end{abstract}

Received March 6, 2013

Accepted May 17, 2013.

${ }^{1}$ Corresponding author: elizabeth.ross@depi.vic.gov.au a significant enrichment for associations with methane production. This suggests that these methane-mitigating diets have altered the rumen microbiome toward naturally low methane-emitting microbial profiles. The contig sequences are predominantly new and include Faecalibacterium spp. The contigs we have identified here are potential biomarkers for low-methane-emitting cattle.

Key words: rumen metagenome, microbiome, sequencing, methane

\section{INTRODUCTION}

Methane $\left(\mathrm{CH}_{4}\right)$, a potent greenhouse gas, is a byproduct of bacterial digestion in ruminants. Most methane is produced in the rumen (87\%) and a small amount $(13 \%)$ is produced in the large intestine (Murray et al., 1976). Enteric methane emissions from farmed ruminant livestock account for approximately $15 \%$ of global methane emissions (Lassey et al., 1997; IPCC, 2007); additionally, a negative relationship exists between enteric methane production and the feed conversion efficiency of an animal (Hegarty et al., 2007). These issues make understanding of the rumen microbes that produce methane of high importance.

The process of digestion in ruminants involves symbiosis between the ruminant and the microorganisms living within its gut. Microorganisms, including bacteria, archaea, fungi, and protozoa, inhabit the rumen of cattle (Dehority, 2003). The collection of these microorganisms is often termed the microbiome, and the DNA extracted from a microbiome sample is termed a metagenome. The methanogens (domain: Archaea) convert the $\mathrm{H}_{2}$ and $\mathrm{CO}_{2}$ to $\mathrm{CH}_{4}$ using a 7-step enzymatic pathway (Attwood and McSweeney, 2008). This provides the methanogens with an energy source and also reduces the $\mathrm{H}_{2}$ concentration in the rumen. Methane is removed from the rumen by eructation to the atmosphere.

Methods based on DNA are attractive for investigations into rumen microbial communities, as culture- 
dependent methods only detect a small proportion of microbial species present in the rumen (Whitford et al., 1998). Until recently, DNA-based methods routinely focused on the $16 \mathrm{~S}$ genes present in metagenomes (e.g., Fernando et al., 2010; Frey et al., 2010). Despite the inherent biases of 16S-based methods, such as unequal amplification in PCR (Hong et al., 2009; Huber et al., 2009; Engelbrektson et al., 2010), they have proven to be a useful and widely used tool for metagenomic analysis. Many metagenomic studies have used 16S-based methods to investigate changes in the rumen microbiome due to different diets (Fernando et al., 2010; Pitta et al., 2010; de Menezes et al., 2011; Stiverson et al., 2011; Hernandez-Sanabria et al., 2012), and have successfully identified large differences in microbiomes as a result of different diets. With current rapid increases in sequencing output, massively parallel sequencing (MPS) can now be applied to the investigation of the rumen microbiome. Whereas MPS solves some problems related to $16 \mathrm{~S}$ studies (e.g., primer bias), it introduces a new complexity in the analysis of microbiome samples.

To date, only a handful of studies have used MPS to investigate the rumen microbiome. The first of these was by Brulc et al. (2009), which gave a functional comparison of both fiber-adherent and liquid fraction microbes, and produced a total of $104 \mathrm{Mbp}$ of sequence data. Hess et al. (2011) later produced $268 \mathrm{Gbp}$ of rumen microbe sequence data from the fiber-adherent rumen fraction. Untargeted sequencing of rumen microbes from the liquid fraction has been used to show that repeatable (by cow) microbial profiles can be obtained using untargeted sequence data (Ross et al., 2012). Although, a the number of studies using untargeted metagenomics to investigate the foregut microbiome of various species is growing (Dai et al., 2012; Pope et al., 2012; Singh et al., 2012; Bhatt et al., 2013), no studies have used untargeted MPS (whole metagenome sequencing) to investigate changes in the rumen microbial population due to dietary additives.

In the current study, we report the effect of 2 methane-mitigating feed additives on the rumen microbial community. Rather than focusing on individual organisms within the rumen, we used untargeted (shotgun), massively parallel sequencing on the Illumina HiSeq2000 platform (Illumina, San Diego, CA) to generate quantitative microbiome profiles. We investigated the hypotheses that (1) the rumen microbiome profiles have been substantially altered by these diets, (2) both diets have altered the metagenomes in the same way, (3) these diets have also had an effect on the microbiota of the hindgut, and (4) markers (in the form of microbial contigs) that are significantly altered by these diets may be possible biomarkers for naturally low methaneproducing animals.

\section{MATERIALS AND METHODS}

\section{Experimental Design}

Samples of rumen fluid and feces from 2 experiments were sequenced, without targeted amplification, on the HiSeq2000 (Illumina; Table 1). The first experiment involved 8 rumen-fistulated Australian Holstein dairy cattle fed either a control diet or a diet with added fat and tannin. This fat and tannin (FT) study was a cross-over design where animals were fed the treatment or control diet for $2 \mathrm{wk}$. Methane production was then measured in a respiration chamber (Grainger et al., 2007) over $2 \mathrm{~d}$, following which samples of rumen fluid (via the fistulae) and feces were collected. The animals were then fed the alternative diet for 2 wk and rumen fluid and feces again sampled after methane measurement. The second experiment involved 32 Australian Holstein dairy cattle fed either a control diet or a diet with added grapemarc. This grapemarc (GMC) study involved 2 forms of the treatment diet (dry and wet grapemarc). Animals were fed their allocated diet for 2 wk, after which methane production was measured using the sulfur hexafluoride tracer technique (Grainger et al., 2007; details in Moate et al., 2012) and rumen fluid was sampled via stomach pump. The GMC diets lowered methane production $(\mathrm{g} / \mathrm{kg}$ of DMI) by approximately $20 \%$, whereas the FT diet lowered methane production $(\mathrm{g} / \mathrm{kg}$ of $\mathrm{DMI})$ by approximately 6\% (Moate et al., 2012; P. J. Moate, unpublished data). After collection, all microbiome samples (rumen fluid and feces) were frozen and stored at $-20^{\circ} \mathrm{C}$ until used.

Changes in the rumen and fecal microbiomes due to these 2 diets were compared and contrasted to gain insight into how the rumen microbiome changes when methane is reduced via dietary additives. In total, 3 comparisons were made, 2 in the FT experiment (rumen and feces) and 1 in the GMC experiment (rumen only).

\section{Diets}

Animals were fed the treatment or control diet for 2 wk before rumen sampling. In addition to the base diet of $6.0 \mathrm{~kg}$ of DM of concentrate (4.1 kg of DM crushed wheat, $1.5 \mathrm{~kg}$ of DM cold-pressed canola meal, $0.12 \mathrm{~kg}$ of DM mineral mix, and $0.28 \mathrm{~kg}$ of DM palabind molasses powder) and ad libitum lucerne hay; the treatment diet of the FT study had $800 \mathrm{~g}$ of cottonseed oil and $400 \mathrm{~g}$ of raw tannin from Acacia mearnsii added to the rumen though the fistulae each day. The control group in the FT experiment had $800 \mathrm{~mL}$ of water added though the fistulae each day. In the GMC experiment (Moate et al., 2012), individual cows were fed each day 
Table 1. Sample characteristics and proportion of reads that align to 3 databases

\begin{tabular}{|c|c|c|c|c|c|c|}
\hline Cow ID & Diet & Sample & $\underset{\mathrm{n}}{\text { Read pairs },{ }^{1}}$ & $\underset{\%}{\text { DPI_rumen }}{ }^{2}$ & $\underset{\%}{\text { JGI_rumen }}{ }^{3}$ & $\begin{array}{l}\text { Combined } \\
\text { rumen, }{ }^{4} \%\end{array}$ \\
\hline 2202 & $\mathrm{Con}_{\mathrm{FT}}{ }^{5}$ & Feces $^{6}$ & $4,686,241$ & 0.92 & 0.22 & 1.03 \\
\hline 2202 & $\mathrm{Con}_{\mathrm{FT}}$ & Rumen $^{6}$ & $5,139,308$ & 4.86 & 1.72 & 6.30 \\
\hline 2202 & $\mathrm{FT}^{7^{\mathrm{TI}}}$ & Feces & $4,839,645$ & 1.94 & 0.28 & 2.08 \\
\hline 2202 & $\mathrm{FT}$ & Rumen & $6,390,148$ & 6.37 & 0.98 & 7.10 \\
\hline 3063 & $\mathrm{Con}_{\mathrm{FT}}$ & Feces $^{6}$ & $8,112,109$ & 1.15 & 0.25 & 1.29 \\
\hline 3063 & $\mathrm{Con}_{\mathrm{FT}}$ & Rumen $^{6}$ & $5,938,570$ & 4.55 & 1.05 & 5.40 \\
\hline 3063 & FT & Feces & $7,581,211$ & 1.46 & 0.34 & 1.63 \\
\hline 3063 & $\mathrm{FT}$ & Rumen & $6,051,276$ & 6.13 & 1.35 & 7.04 \\
\hline 6803 & $\mathrm{Con}_{\mathrm{FT}}$ & Feces $^{6}$ & $9,676,293$ & 1.05 & 0.23 & 1.16 \\
\hline 6803 & $\mathrm{Con}_{\mathrm{FT}}$ & Rumen $^{6}$ & $5,900,689$ & 4.89 & 1.19 & 5.87 \\
\hline 6803 & FT & Feces & $6,706,552$ & 1.26 & 0.28 & 1.39 \\
\hline 6803 & FT & Rumen & $5,739,286$ & 4.50 & 0.98 & 5.24 \\
\hline 6838 & $\mathrm{Con}_{\mathrm{FT}}$ & Feces & $6,895,378$ & 1.44 & 0.28 & 1.61 \\
\hline 6838 & FT & Feces & $5,221,265$ & 2.06 & 0.38 & 2.26 \\
\hline 6838 & $\mathrm{FT}$ & Rumen & $6,121,980$ & 6.29 & 1.31 & 7.21 \\
\hline 6852 & $\mathrm{Con}_{\mathrm{FT}}$ & Feces $^{6}$ & $5,871,457$ & 1.56 & 0.25 & 1.68 \\
\hline 6852 & $\mathrm{Con}_{\mathrm{FT}}$ & Rumen $^{6}$ & $5,829,005$ & 7.47 & 1.01 & 8.20 \\
\hline 6852 & $\mathrm{FT}$ & Feces & $7,855,120$ & 1.35 & 0.34 & 1.55 \\
\hline 6852 & FT & Rumen & $4,832,105$ & 5.66 & 0.82 & 6.26 \\
\hline 6859 & $\mathrm{Con}_{\mathrm{FT}}$ & Feces $^{6}$ & $4,095,059$ & 1.84 & 0.34 & 2.05 \\
\hline 6859 & $\mathrm{Con}_{\mathrm{FT}}$ & Rumen $^{6}$ & $5,838,894$ & 4.80 & 1.31 & 5.88 \\
\hline 6859 & FT & Feces & $5,602,536$ & 2.37 & 0.32 & 2.53 \\
\hline 6859 & FT & Rumen & $5,245,035$ & 6.68 & 1.06 & 7.39 \\
\hline 6882 & $\mathrm{Con}_{\mathrm{FT}}$ & Feces $^{6}$ & $5,740,622$ & 1.35 & 0.29 & 1.51 \\
\hline 6882 & $\mathrm{Con}_{\mathrm{FT}}$ & Rumen $^{6}$ & $6,076,296$ & 3.95 & 0.95 & 4.74 \\
\hline 6882 & $\mathrm{FT}$ & Feces & $5,191,205$ & 1.98 & 0.42 & 2.23 \\
\hline 6882 & FT & Rumen $a^{8}$ & $5,832,342$ & 3.49 & 0.78 & 4.06 \\
\hline 6882 & FT & Rumen $b^{8}$ & $4,870,694$ & 4.57 & 1.01 & 5.32 \\
\hline 7920 & $\mathrm{Con}_{\mathrm{FT}}$ & Feces $^{6}$ & $4,769,719$ & 1.82 & 0.27 & 1.99 \\
\hline 7920 & $\mathrm{Con}_{\mathrm{FT}}$ & Rumen $^{6}$ & $3,973,810$ & 8.01 & 1.42 & 9.02 \\
\hline 7920 & FT & Feces & $5,109,024$ & 1.62 & 0.31 & 1.80 \\
\hline 7920 & $\mathrm{FT}$ & Rumen & $6,044,055$ & 4.91 & 1.20 & 5.80 \\
\hline 1995 & $\mathrm{Con}_{\mathrm{G}}{ }^{9}$ & Rumen & $8,570,618$ & 8.83 & 1.87 & 10.20 \\
\hline 2028 & $\mathrm{Con}_{\mathrm{G}}$ & Rumen & $9,530,428$ & 9.04 & 1.93 & 10.44 \\
\hline 2042 & $\mathrm{Con}_{\mathrm{G}}$ & Rumen & $8,795,725$ & 6.05 & 2.37 & 8.02 \\
\hline 2165 & $\mathrm{Con}_{\mathrm{G}}$ & Rumen & $6,994,291$ & 6.77 & 2.44 & 8.75 \\
\hline 3060 & $\mathrm{Con}_{\mathrm{G}}$ & Rumen & $10,199,248$ & 8.63 & 1.92 & 10.04 \\
\hline 4570 & $\mathrm{Con}_{\mathrm{G}}$ & Rumen & $13,438,217$ & 8.08 & 2.12 & 9.69 \\
\hline 5679 & $\mathrm{Con}_{\mathrm{G}}$ & Rumen & $5,385,519$ & 7.05 & 2.49 & 9.07 \\
\hline 657 & $\mathrm{Con}_{\mathrm{G}}$ & Rumen & $5,411,824$ & 6.92 & 2.50 & 8.96 \\
\hline 6833 & $\mathrm{Con}_{\mathrm{G}}$ & Rumen & $7,192,530$ & 7.71 & 1.94 & 9.17 \\
\hline 6857 & $\mathrm{Con}_{\mathrm{G}}$ & Rumen & $5,537,990$ & 8.02 & 2.10 & 9.63 \\
\hline 6870 & $\mathrm{Con}_{\mathrm{G}}$ & Rumen & $10,111,427$ & 8.86 & 2.06 & 10.41 \\
\hline 7939 & $\mathrm{Con}_{\mathrm{G}}$ & Rumen & $6,459,344$ & 7.64 & 1.81 & 8.98 \\
\hline 1990 & $\operatorname{Dry}_{G}{ }^{10}$ & Rumen & $7,069,477$ & 6.60 & 2.17 & 8.35 \\
\hline 2196 & $\operatorname{Dry}_{G}$ & Rumen & $5,691,296$ & 7.65 & 2.17 & 9.35 \\
\hline 2204 & Dry $_{G}$ & Rumen & $6,726,286$ & 5.98 & 2.00 & 7.60 \\
\hline 2209 & Dry $_{G}$ & Rumen & $4,246,828$ & 7.33 & 2.14 & 9.01 \\
\hline 3082 & $\operatorname{Dry}_{G}$ & Rumen & $6,083,539$ & 6.77 & 2.22 & 8.56 \\
\hline 4522 & Dry $_{G}$ & Rumen & $7,190,784$ & 8.13 & 1.88 & 9.52 \\
\hline 5592 & $\operatorname{Dry}_{G}$ & Rumen & $4,721,060$ & 6.13 & 2.19 & 7.94 \\
\hline 5630 & Dry $_{G}$ & Rumen & $6,632,937$ & 6.92 & 2.41 & 8.88 \\
\hline 5660 & Dry $_{G}$ & Rumen & $7,101,599$ & 6.67 & 2.16 & 8.40 \\
\hline 6823 & Dry $_{G}$ & Rumen & $10,873,783$ & 5.83 & 1.96 & 7.44 \\
\hline 1912 & $\mathrm{Wet}_{\mathrm{G}}{ }^{11}$ & Rumen & $6,360,807$ & 5.37 & 2.51 & 7.52 \\
\hline 2032 & Wet $_{G}$ & Rumen & $6,490,865$ & 6.76 & 1.87 & 8.28 \\
\hline 2074 & Wet $_{G}$ & Rumen & $4,539,275$ & 6.12 & 1.87 & 7.64 \\
\hline 2141 & Wet $_{G}$ & Rumen & $6,928,120$ & 6.07 & 2.27 & 7.99 \\
\hline 3097 & Wet $_{G}$ & Rumen & $4,129,699$ & 7.14 & 2.24 & 8.95 \\
\hline 4567 & Wet $_{\mathrm{G}}$ & Rumen & $7,145,390$ & 6.64 & 2.50 & 8.73 \\
\hline 6747 & Wet $_{G}$ & Rumen & $6,978,648$ & 6.38 & 2.18 & 8.16 \\
\hline 6810 & Wet $_{G}$ & Rumen & $6,148,444$ & 7.10 & 1.97 & 8.65 \\
\hline
\end{tabular}


Table 1 (Continued). Sample characteristics and proportion of reads that align to 3 databases

\begin{tabular}{|c|c|c|c|c|c|c|}
\hline Cow ID & Diet & Sample & $\underset{\mathrm{n}}{\operatorname{Read}}$ pairs, ${ }^{1}$ & $\underset{\%}{\text { DPI_rumen }}{ }^{2}$ & JGI_rumen ${ }^{3}$ & $\begin{array}{l}\text { Combined } \\
\text { rumen, }{ }^{4} \%\end{array}$ \\
\hline 6853 & Wet $_{\mathrm{G}}$ & Rumen & $4,829,180$ & 6.69 & 1.76 & 8.06 \\
\hline 7354 & Wet $_{\mathrm{G}}$ & Rumen & $6,306,769$ & 4.44 & 2.26 & 6.46 \\
\hline \multicolumn{7}{|c|}{${ }^{1}$ Number of read pairs remaining after trimming (removal of poor quality sequence). } \\
\hline \multicolumn{7}{|c|}{${ }^{2}$ DPI_rumen is a database of contigs generated from rumen fluid microbes (Ross et al., 2012). } \\
\hline \multicolumn{7}{|c|}{${ }^{3}$ JGI_rumen is a database of contigs generated from fiber-adherent rumen microbes (Hess et al., 2011). } \\
\hline \multicolumn{7}{|c|}{${ }^{4}$ Combined rumen is the 2 databases combined (DPI_rumen and JGI_rumen). } \\
\hline \multicolumn{7}{|c|}{${ }^{5} \mathrm{Con}_{\mathrm{FT}}=$ control diet for the fat and tannin experiment. } \\
\hline \multicolumn{7}{|c|}{${ }^{6}$ These sequence sets are published in Ross et al. (2012). } \\
\hline \multicolumn{7}{|c|}{${ }^{7} \mathrm{FT}=$ treatment diet of the fat and tannin experiment. } \\
\hline \multicolumn{7}{|c|}{$\begin{array}{l}{ }^{8} \text { The a and b designations following the sample type indicate technical replicates of the same sample, wit } \\
\text { separate DNA extractions. }\end{array}$} \\
\hline \multicolumn{7}{|c|}{${ }^{9} \mathrm{Con}_{\mathrm{G}}=$ control diet for the grapemarc experiment. } \\
\hline \multicolumn{7}{|c|}{${ }^{10}$ Dry $_{\mathrm{G}}=$ dry supplement treatment diet of the grapemarc experiment. } \\
\hline & & & & & & \\
\hline
\end{tabular}

on a control diet that contained approximately $4.0 \mathrm{~kg}$ of DM of crushed wheat, $0.2 \mathrm{~kg}$ of DM of molasses, 0.1 $\mathrm{kg}$ of DM of minerals, and $14 \mathrm{~kg}$ of DM of alfalfa hay. Cows in the GMC treatments groups were individually fed the control diet, but $5 \mathrm{~kg}$ of $\mathrm{DM} / \mathrm{d}$ of either dry crumbled or ensiled grapemarc was substituted for an equal quantity of alfalfa hay.

\section{Molecular Techniques}

The DNA was extracted from all microbiome samples using the PowerMax Soil DNA Isolation kit (MoBio, Carlsbad, CA). Libraries were then prepared on the DNA extract using an in-house library preparation protocol, which included 2 levels of indexing (1 third read and 1 internal index on read 2). Libraries were then sequenced on the HiSeq2000 (Illumina) on $101 \mathrm{bp}$ paired end runs. All sequences presented in this publication can be obtained from the MG-RAST server (http://metagenomics.anl.gov/; project ID:4126 Bovine Metagenome).

\section{Sequence Quality Control}

Poor quality sequence was removed from the data set based on the Phred quality score of the nucleotide. The Phred quality score is defined as $\mathrm{Q}$, where $\mathrm{Q}=-10$ $\log _{10}$ (probability of a base calling error). Bases from the $5^{\prime}$ end of each read were removed until a maximum of 3 bases remained with a $\mathrm{Q}$ of $<15$. If the read was $<50$ bp long after this trimming, or the average $\mathrm{Q}$ of the read was $<20$, it was discarded. The remaining data was decloned using kmers_remove_clonal from libngs (https://github.com/sylvainforet/libngs). Eukaryotic contamination was not removed from the data set.

\section{Metagenome Profiles}

Alignments of sequence reads were to contig (genome fragment) databases using Burrows-Wheeler Aligner (BWA)-backtrack (Li and Durbin, 2009) with all default settings except of the -o0 and -e0 flags, which suppress gap opening, and extension in the aln command. The contig databases were both derived from the rumen of cattle. The first (Ross et al., 2012) was from Roche 454 sequencing of rumen fluid from lactating Holstein cattle located at the sample location as our samples but sampled several years earlier. The second was an assembly of Illumina sequencing of fiber-adherent rumen microbes (Hess et al., 2011). These 2 contig databases were combined and referred to as the rumen database; BWA returns only 1 alignment per sequence. Alignments were then converted into a matrix of sample ID versus contig, which contained the number of reads that mapped to each contig from each sample, using $R$ statistical package (R Development Core Team, 2011). This matrix was termed the rumen metagenome profile matrix.

\section{Diet Effect on Metagenome Profiles}

Modeling was performed on the natural log of the alignment matrix. To account for cells in the matrix with a value of zero, 0.01 was added to each cell before log-transformation. Diet was fitted as a fixed effect and cow and contig were both fitted as random effects. A linear mixed model $\left(Y_{i j k}=\mu+\operatorname{diet}_{i}+\operatorname{contig}_{j}+\operatorname{animal}_{k}\right.$ $\left.+e_{i j k}\right)$ was fitted to the read count data using ASReml (Gilmour et al., 2006), where $Y_{i j k}=\log _{\mathrm{n}}$ transformed counts, $\mu=$ the overall mean; $\operatorname{diet}_{i}=$ the treatment or control diet $i$ that the animal had been fed before 
sampling, contig $_{j}=$ the contig $j$ in the reference database, animal $_{k}=$ the host animal $k$ that samples were taken from (omitted for analysis of the GMC study), and $e_{i j k}=$ unexplained variance. The significance of the fixed effects was assessed using Wald F statistics. The significance of the random effects was assessed using log-likelihood.

\section{Hierarchical Clustering of Metagenome Profiles}

Hierarchical clustering was performed on the rumen metagenome profile matrix using $\mathrm{R}$ ( $\mathrm{R}$ Development Core Team, 2011; hclust command: $\operatorname{method}=$ Ward; dist command: method = Canberra). The relationship between diet and the subtree that the sample was nested in was assessed using Fisher's exact test. The GMC experiment showed animal 7354 as an outlier (it formed a third subtree of its own), this sample was removed from the statistical analysis between subtree and dietary additive. Inclusion of animal 7354 in either of the GMC groups does not affect the significance conclusion at the $P<0.05$ level.

\section{Metagenomic Predictions from Metagenome Profiles}

Metagenomic predictions (Ross et al., 2013) were performed to investigate the hypothesis that we can predict the type of diet a cow is being fed (methane mitigating or not methane mitigating) from the microbiome alone. Metagenomic profiles (Ross et al., 2012) were used to create metagenomic relationship matrices. A metagenomic profile is the vector of counts of MPS shotgun reads that align to each contig in a database; BWA (Li and Durbin, 2009) was used to perform alignments. Several metagenomic profiles were combined to form an $n \times m$ matrix $\mathbf{X}$ with elements $x_{i j}$, the $\log$ transformed and standardized count for sample $i$ for contig $j$, with $n$ samples and $m$ contigs. These profiles were then compared to make a rumen microbiome relationship matrix (calculated as $\mathbf{G}=\mathbf{X X}^{\prime} / m$ ). Best linear unbiased prediction (Henderson, 1984) was then used to predict phenotypes for validation samples. A mixed model was fitted to the data: $\mathbf{y}=\mathbf{1}_{n} \mu+\mathbf{Z g}+$ $\mathbf{e}$, where $\mathbf{y}$ is a vector of diets ( 1 for methane-mitigating diet, 0 for control diet) with 1 record per sample; $\mathbf{1}_{n}$ is a vector of ones; $\mu$ is the overall mean; $\mathbf{Z}$ is a design matrix allocating records to samples; $\mathbf{g}$ is a random effect estimate $\sim N\left(0, \mathbf{G} \sigma_{g}^{2}\right)$; and $\mathbf{e}=$ unexplained variance. Using ASReml (Gilmour et al., 2006), phenotype of the samples $(\hat{g})$ were predicted as

$$
\left[\begin{array}{c}
\hat{\mu} \\
\hat{g}
\end{array}\right]=\left[\begin{array}{cc}
\mathbf{1}_{n}{ }^{\prime} \mathbf{1}_{n} & \mathbf{1}_{n}{ }^{\prime} \mathbf{Z} \\
\mathbf{Z}^{\prime} 1_{n} & \mathbf{Z}^{\prime} \mathbf{Z}+\mathbf{G}^{-1} \frac{\sigma_{e}^{2}}{\sigma_{g}^{2}}
\end{array}\right]^{-1}\left[\begin{array}{c}
\mathbf{1}_{n}{ }^{\prime} \mathbf{y} \\
\mathbf{Z}^{\prime} \mathbf{y}
\end{array}\right] .
$$

Nine predictions were performed in total. Phenotypes from each subexperiment (GMC, FT rumen, and FT feces) were first predicted from a subset of the same data set. Across data set predictions were then carried out, such that each of GMC, FT rumen, and FT feces was predicted using each of the alternative 2 data sets as a reference (e.g., GMC was predicted from FT feces, then GMC predicted from FT rumen and vice versa).

Where multiple samples were available from each cow (as in the FT study), all samples from the animal undergoing prediction were removed from the reference population. For the prediction, which used the GMC study as both reference and test population, half of the animals were used in the reference population, whereas the other half made up the test population. For across experiment predictions (FT from GMC and vice versa), all relevant samples were included in the reference population.

\section{Differential Representation of Contigs Between Control and Methane-Mitigating Diets}

The rumen metagenome profile matrix of each experiment (FT rumen, FT feces, and GMC) was used to assess which contigs were differentially represented between controls and treatments. Contigs with an average of 1 or fewer reads per sample were removed from each experiment matrix. Differentially represented contigs between treatments were extracted from the alignment matrix using edgeR (Robinson and Smyth, 2007; Robinson and Smyth, 2008; Robinson et al., 2010) and a GLM with tag-wise dispersion $(P<0.01)$. EdgeR is a software originally developed for gene expression data analysis. Contigs differentially represented in multiple experiments were then assigned to taxa using BLASTn (nucleotide BLAST, a program for comparing DNA sequences to a nucleotide database; run on an internal system, last updated June 2012). All BLASTn results were passed to MEGAN4 (MEtaGenome ANalyzer, a program for analyzing metagenome samples; Huson et al., 2007, 2011) for interpretation.

The KEGG pathways (Kyoto Encyclopedia of Genes and Genomes; http://www.genome.jp/kegg/) are a collection of manually maintained pathway maps that represent knowledge of the molecular interaction and reaction between gene functions. Here we have used them to assign the sequence reads to a biological function. To achieve this, 100,000 reads from each sample (all with average $\mathrm{Q}$ scores of 30 and no bases with a Q score below 15) were used for the BLAST analysis. The BLASTn and BLASTx (a program for comparing DNA sequences to a protein database) programs were performed on an internal system to nt (nucleotide) and $\mathrm{nr}$ (nonredundant protein) databases (both last up- 
dated in June 2012). The MEGAN4 program (Huson et al., 2007; Huson et al., 2011) was used to extract the KEGG pathway assignments from the BLASTx results.

The KEGG second-level pathways were used to accesses functional changes in the metagenomes; KEGG second-level pathways with fewer than 1,000 total reads assigned to them were not analyzed. Assignments were converted to percentage of assigned reads, such that the assignments of each sample totaled 100\%. Differential representation of KEGG pathways was then assessed on the percentages using a $t$-test. The amount of overlap between experiments was tested for significance using cumulative hypergeometric probability.

\section{Association Between Individual Contig Counts and Methane Production}

The relationship between contigs abundance and residual methane production was assessed. This analysis used a validation set of 16 independent animals, which were fed a control diet for a minimum of 2 wk. After 2 wk, methane production was measured by respiration chamber (Grainger et al., 2007) over 2 d before rumen fluid was sampled via stomach pump. The residual methane production of each animal in the validation set, in which all animals were fed the same diet, was calculated as $\mathrm{CH}_{4}$ corrected for DMI, that is, the average residual methane production for that animal when a linear model was fitted $\left(\mathrm{CH}_{4}\right.$ production $\left.\sim \mathrm{DMI}\right)$. This was done to remove the large effect of DMI from the methane production value.

The MASS package (Venables and Ripley, 2002) in $\mathrm{R}$ was used to fit a model (reads mapping to contig $\sim$ residual methane production). A negative binomial distribution was used for the model. Negative binomial linear models are suitable for count data such as this due to the over-dispersed nature of massively parallel sequencing count data, and are recommended for use in RNAseq analysis that have similar objectives and data structure to this analysis (e.g., Robinson and Smyth, 2007, 2008; Robinson et al., 2010; Sun, 2012).

\section{RESULTS}

\section{Comparison of Sequence Reads to Other Rumen Sequence Databases}

To assess the amount of overlap between our MPS data and other databases we first compared our sequencing reads to publically available data sets. The MPS reads from the Illumina HiSeq2000 were aligned to 2 databases (Table 1 ) that were used in an earlier study (Ross et al., 2012). Rumen fluid samples had the highest alignment rate to the DPI_rumen database (6.49\%), which was made using DNA from bovine rumen fluid. A smaller amount aligned to the JGI_rumen database (1.80\%), obtained from Hess et al. (2011) and was generated from fiber-adherent microbes in the bovine rumen. An almost (95\%) complete independence of the reads mapping to each rumen database was observed when the 2 databases were combined. Because of this, for subsequent analysis, we combined these 2 rumen databases (DPI_rumen and JGI_rumen), which are hence forth referred to as the rumen database.

To compare the amount of sequence that was similar to already published sequences, 100,000 reads were compared with the nt database using BLASTn. For the rumen and feces samples, 35.4 and $35.2 \%$ of reads, respectively, had at least 1 hit with a very liberal maximum e-value $(<0.02$; i.e., $2 \%$ of reads are expected to align to the database by chance), suggesting that well over half of the DNA fragments produced are novel when compared with these widely used databases.

\section{Effect of Diet on Rumen Metagenome Profiles}

We tested the hypothesis that the methane-mitigating diets had a significant effect on the rumen microbial

Table 2. Linear mixed model analysis of the 3 sample sets: rumen samples from the grapemarc experiment (GMC), rumen samples from the fat and tannin experiment (FT rumen), and feces from the fat and tannin experiment $(\text { FT feces })^{1}$

\begin{tabular}{lccc}
\hline Item & GMC & FT rumen & FT feces \\
\hline Diet effect, $P$-value (fixed) & $<0.001$ & 0.001 & $<0.001$ \\
Animal effect, \% (random) & - & $0.56^{*}$ & $1.82^{*}$ \\
Contig effect, \% (random) & $39.79^{*}$ & $23.93^{*}$ & $26.01^{*}$ \\
Error, \% & $60.21^{*}$ & $75.51^{*}$ & $72.17^{*}$ \\
\hline
\end{tabular}

${ }^{1}$ A linear mixed model $\left(Y_{i j k}=\mu+\operatorname{diet}_{i}+\operatorname{contig}_{j}+\operatorname{animal}_{k}+e_{i j k}\right)$ was fitted to the sample by contig matrix using ASReml (Gilmour et al., 2006), where $Y_{i j k}=$ counts, $\mu=$ the mean, $\operatorname{diet}_{\mathrm{i}}=$ the diet the animal was on at the time of sampling, $\operatorname{animal}_{k}=$ the host animal that samples were taken from (omitted for the GMC study), $\operatorname{contig}_{j}=$ the contig in the database that the reads aligned to, and $e=$ unexplained variance. Significance of random effects was calculated by likelihood ratio tests.

$* P<0.0001$. 


\section{a) Grapemarc}

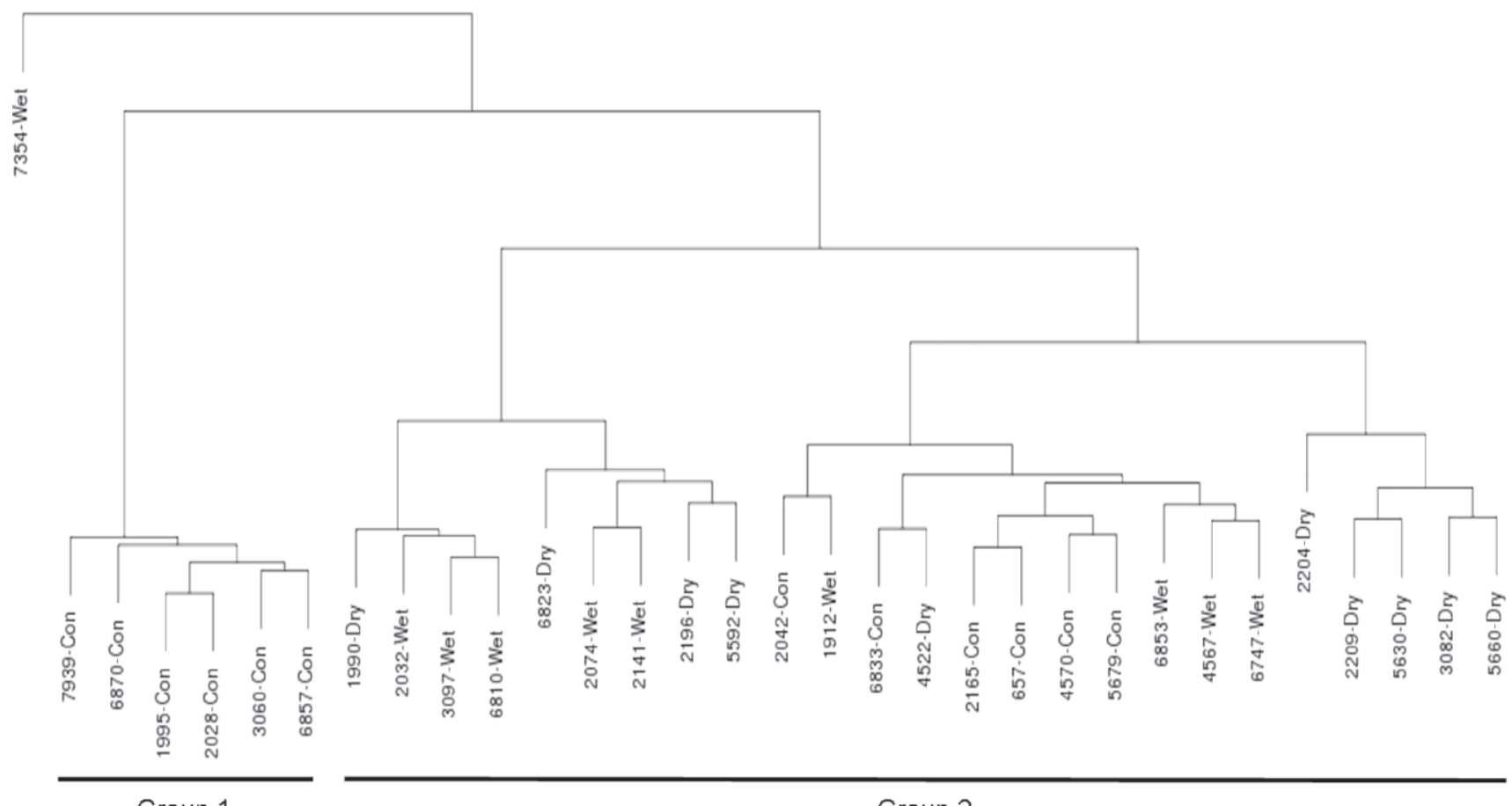

Group 1

Group 2

\section{b) Fat and Tannin Rumen}

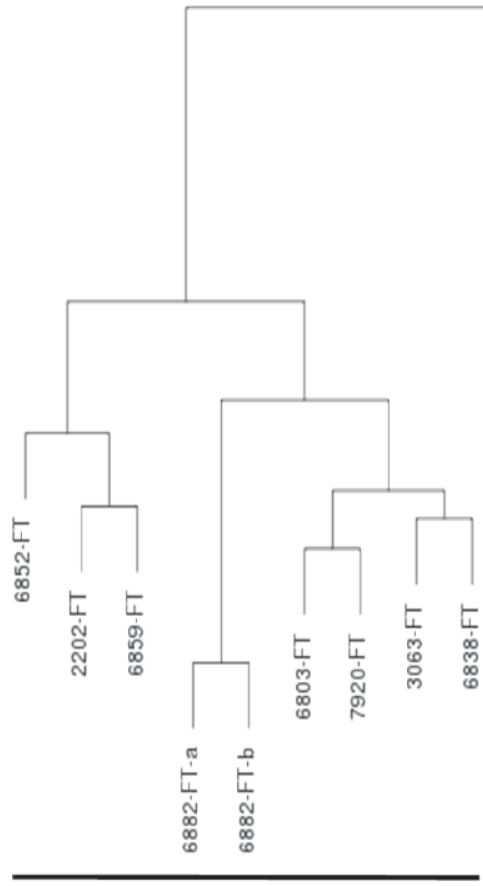

Group 1

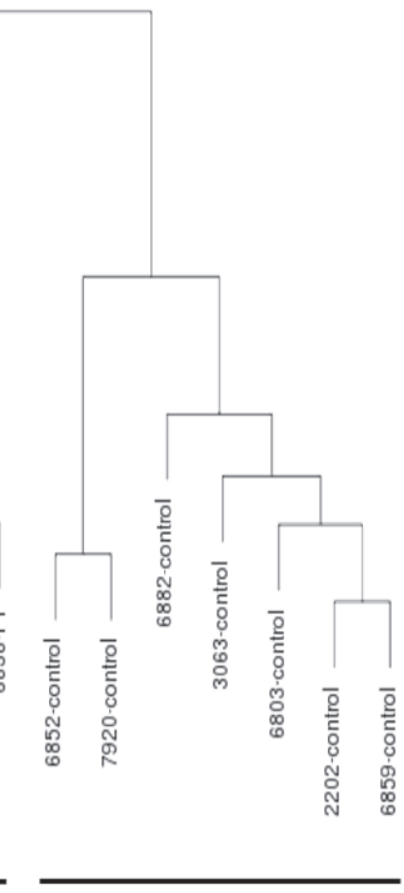

Group 2

\section{c) Fat and Tannin Feces}

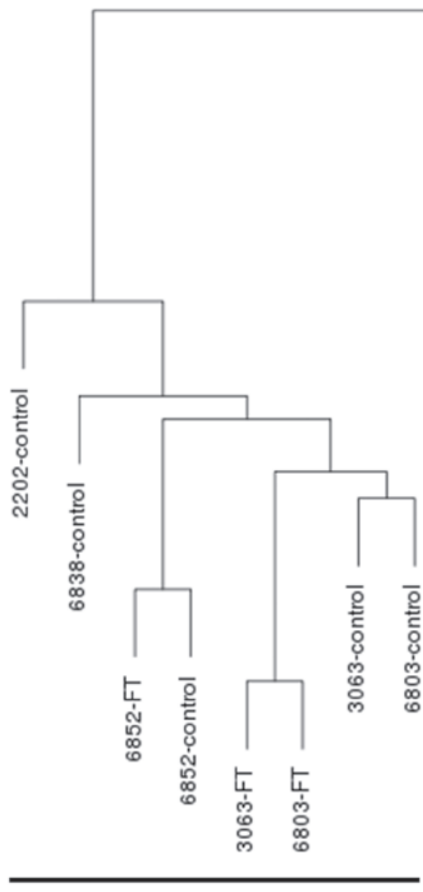

Group 1

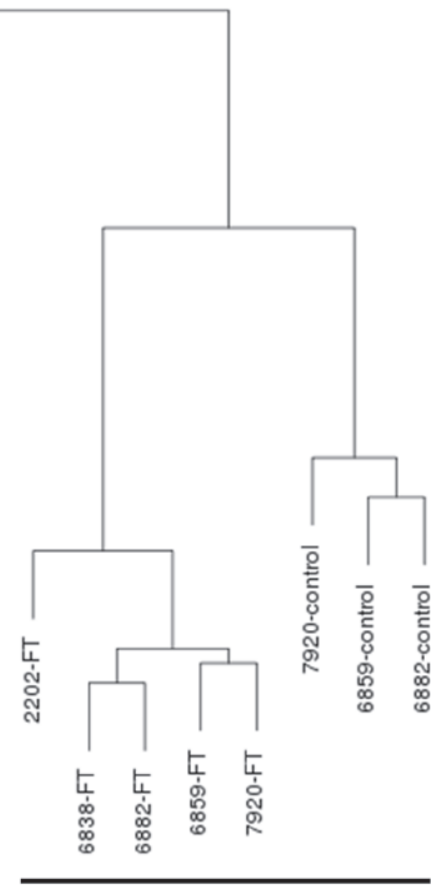

Group 2

Figure 1. Hierarchical clustering of microbiome samples. A normalized number of reads was mapped to the combined rumen database. The number of reads mapping to each contig in the database was used to make a sample by contig matrix that contained counts of the number of reads mapping to each contig from each sample. Hierarchical clustering was performed on the matrix using the $\mathrm{R}$ statistical package ( $\mathrm{R}$ Development Core Team, 2011; hclust command, method = Ward; dist command, method = Canberra). 
profiles by first aligning 3.66 million reads from each sample to the rumen database. We then used a linear mixed model with the number of reads that mapped to each contig in the database from each sample as the $y$ variable, a fixed effect of diet and random effect of animal and contig. The model was fitted for each experiment separately. Diet had a significant effect for both experiments (Table 2). These modeling results confirm that the diets have indeed altered the rumen microbiomes. Contig also had a large effect in the model, highlighting how different species (represented here by contigs) have very different abundance patterns within the bovine metagenome. For the FT study, where multiple samples were recorded per animal, the effect of animal (repeatability) on the counts was small (explaining $0.56 \%$ of the variance in rumen and $1.82 \%$ in feces), but significant (Table 2).

After establishing that the diets have a significant effect on the rumen and fecal microbiomes, we further explored the similarities between samples. The metagenomic profiles were used to perform hierarchical clustering. At the deepest separation of the 2 subtrees perfect separation by diet was not observed; however, a significant relationship was observed between diet and the metagenome-based subtree allocation (Fishers exact probability test, 2-tailed; $P=0.0013$ and 0.00016 for GMC and FT rumen, respectively; Figure 1a and b). When the fecal samples were used, no relationship appeared to exist between diet and the metagenomebased subtree allocation (Fisher Exact Probability Test, 2-tailed; $P=0.619$; Figure 1c).

\section{Metagenomic Predictions for Diet}

After observing the strong effect of diet on the rumen microbiome in both experiments via the linear mixed model and hierarchical clustering techniques, we investigated whether the 2 different diet treatments (FT and GMC) had led to similar rumen metagenome profiles. To investigate the hypothesis that the diets had altered the metagenomes in similar ways, we generated a metagenomic relationship matrix from the metagenome profiles and subsequently used it to predict the diet that the animals had been fed (control $=0$, treatment $=1$ ) at the time of sampling using only the microbiome. That is, can we predict the type of diet a cow is being fed (methane mitigating or not methane mitigating) from the microbiome alone?

We first tested if we could predict the diet of an animal's samples using the other samples from that experiment. We were able to successfully predict if an animal had been fed the control or treatment diet using only its microbiome profile $(\mathrm{r}=0.790-0.836$; Figure 2$)$. Next, to assess the similarities between diets we used the alternative experiment as the reference population. We were able to predict the diet that each animal had been fed using the other experiment as a reference, even across sample types (e.g., prediction of GMC rumen data set from the FT feces data set), with an accuracy of $\mathrm{r}=0.634$ to 0.861 (Figure 2). The predicted values for the diet samples were all significantly higher in the treatment compared with the controls ( $t$-test, one-tailed, $P<0.001)$.

\section{Differential Representation Analysis}

After establishing that changes occurred in the samples' microbiomes due to the methane-mitigating dietary additives and obtaining evidence that both diets had altered the microbiomes in similar ways, we examined whether specific species and functional characteristics of the microbiomes were affected by the feed additives. We did this using 2 approaches: first, by identifying differently represented contigs and then assigning them to taxa; and, second, by assigning reads to KEGG pathways and identifying the pathways that are differentially represented between diets.

Many more contigs showed a significant difference between treatment and controls in each experiment than expected by chance (Table 3). We observed 678 contigs with sufficient coverage for a comparison in all 3 data sets. Of the 678 contigs, 43 were differently represented in all 3 comparisons (Figure 3a). The overlap in significant contigs between studies was significantly higher than expected by chance (expected by chance $=$ $\left.1.6, \chi^{2}=1,043, P<0.0001\right)$. The 2 rumen data sets and the 2 FT data sets (rumen and feces) also showed significantly more overlap between them than expected by chance (Figure 3b-c).

To examine whether the significant contigs showed over-representation in certain taxa, we used BLAST to assign the contigs to a taxa and then compared the taxon distribution of all tested contigs to the taxon distribution of the significant contigs. The 43 contigs that were significantly differentially represented in all 3 comparisons were over-represented in Faecalibacterium prausnitzii (identity: 69-79\%; Figure 4 and 5a) and all the higher taxa of F. prausnitzii (Bacteria, Firmicutes, Clostridiales, Ruminococcaceae).

After establishing that large effects of these diets on the species were present in the microbiomes (as observed by assigning contigs to taxa), we investigated the effect that these changes had on the functional aspects of the microbiomes. Reads were assigned to KEGG second level functional pathways and the percentage of reads in each pathway was compared between treatments and control samples. The feces microbiomes did not have any more differences than expected by chance. The FT 


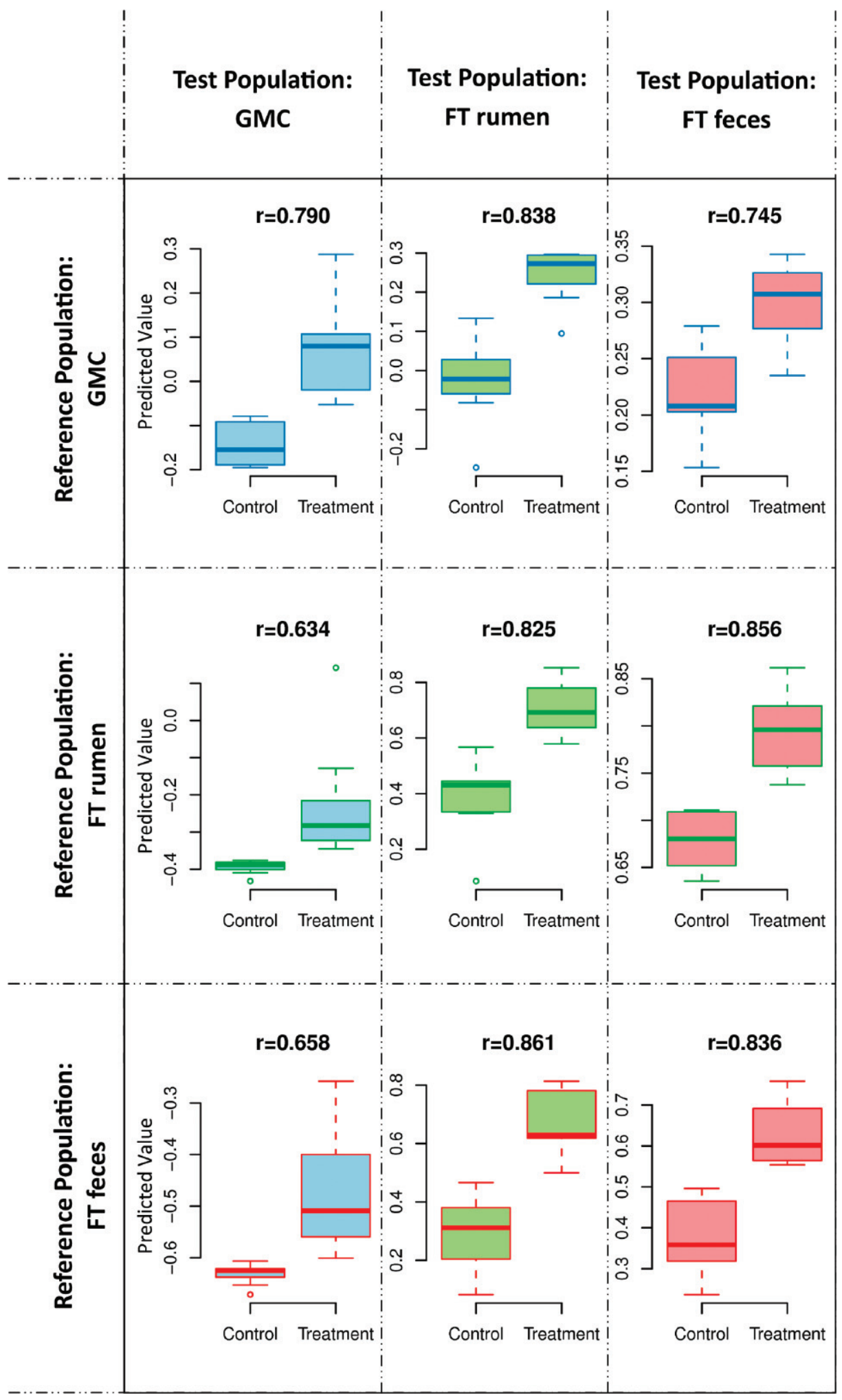

Figure 2. Metagenomic predictions of diet. Using each data set as a reference (from top to bottom: GMC, FT rumen, FT feces) the diet status of the test population (from left to right: GMC, FT rumen, FT feces) was predicted. The microbiome profiles from each sample were used to make a metagenomic relationship matrix. ASReml (Gilmour et al., 2006) was then used to predict the unknown diets of the test population based on the known diets of the reference population and the metagenomic relationship matrix as in Ross et al. (2013). Correlations (r) between the predicted values and the actual diet of the sample (control: 0, treatment: 1 ) indicated the accuracy of the prediction. GMC $=$ grapemarc; $\mathrm{FT}=$ fat and tannin. Color version available in the online PDF. 
Table 3. Significantly differently represented contigs between diets (control versus treatment) $)^{1}$

\begin{tabular}{lccc}
\hline Item $^{2}$ & $\begin{array}{c}\mathrm{GMC}^{3,6} \\
(\mathrm{n}=32)\end{array}$ & $\begin{array}{c}\text { FT rumen } \\
(\mathrm{n}=16)\end{array}$ & $\begin{array}{c}\text { FT feces } \\
(\mathrm{n}=16)\end{array}$ \\
\hline$>10 \times N$ reads, $\mathrm{n}$ & & & \\
$\quad$ Contigs tested & 10,782 & 6,429 & 1,637 \\
$P<0.05$ & $4,236[12.7 \%]$ & $2,355[13.6 \%]$ & $376[21.8 \%]$ \\
$P<0.01$ & $2,086[2.0 \%]$ & $1,659[1.4 \%]$ & $305[1.2 \%]$ \\
$>100 \times N$ reads, $\mathrm{n}$ & 419 & 252 & 77 \\
Contigs tested & $263[8.0 \%]$ & $124[10.2 \%]$ & $10[38.5 \%]$ \\
$P<0.05$ & $156[1.7 \%]$ & $64[1.9 \%]$ & $7[1.4 \%]$ \\
$P<0.01$ & &
\end{tabular}

${ }^{1}$ The $\mathrm{R}$ statistical package ( $\mathrm{R}$ Development Core Team, 2011) and EdgeR (Robinson and Smyth, 2007; Robinson and Smyth, 2008; Robinson et al., 2010) were used to identify contigs significantly associated with the dietary treatments.

${ }^{2}$ Average total reads per contig was greater than the number of samples $(N)$ times the cuttoff (10 or 100). Contigs with less than this number of reads mapping to them from each animal (on average) were not tested. ${ }^{3} \mathrm{GMC}=$ rumen samples from the grapemarc experiment.

${ }^{4} \mathrm{FT}$ rumen $=$ rumen samples from the fat and tannin experiment.

${ }^{5} \mathrm{FT}$ feces $=$ feces from the fat and tannin experiment.

${ }^{6}$ False discovery rate [displayed in brackets] calculated as number of tests $\times P /$ number of significant results $=$ percentage of the significant results that are expected to be due to chance alone.

rumen study showed 7 (false discovery rate $=19 \%$ ) differentially represented pathways $(t$-test, $P<0.05)$, whereas the GMC study showed 8 (false discovery rate $=17 \%$ ) differently represented pathways $(t$-test, $P<$ 0.05 ). Four pathways (amino acid metabolism, carbohydrate metabolism, translation, and biosynthesis of other secondary metabolites) were differently represented in both the FT and GMC studies. Although the overlap between the 2 studies only approached significance [cumulative hypergeometric probability, $\mathrm{P}(\mathrm{X}$ $\geq 4)=0.0596]$, every pathway that was differentially represented in one study showed the same directional change in the second study (Figure 6).

\section{Validation in Independent Animals}

One hypothesis to explain the overlap in the differentially represented contigs between the FT and the GMC studies would be that the 43 contigs identified as significantly differentially represented in all $3 \mathrm{com}-$ parisons are markers for low methane production. To examine this hypothesis we used sequence data from rumen samples from 16 additional Australian Holstein dairy cows, all on a control diet, but with methane emissions measured. Substantial variation was observed in the methane yields and residual methane production of these animals. We created a rumen microbiome profile matrix containing the 678 contigs that had been tested in the 3 diet comparisons. This allowed us to test for an over-representation of contigs that were significantly associated with methane production in the 43 diet-associated contigs compared with the rest of the database. A linear regression was used to estimate the effect of each contig's abundance (as measured by number of reads that map to that contig) on the residual methane production of the animal in the reduced data set. Of the 678 contigs, 48 were significantly associated with residual methane production (negative binomial distribution; $P<0.05$ ).

To test for overlap between the contigs significantly associated with residual methane and contigs differentially represented between the control and treatment samples of the methane-mitigating diets, we applied a cumulative hypergeometric distribution test. This test gives the probability of getting an overlap as large as or larger than what is observed by chance alone. Therefore, we were able to estimate the probability of the overlap between the methane-associated contigs and the diet-associated contigs happening by chance. The overlap between the methane-associated contigs and the diet-associated contigs was highly significant [Figure 3d; $\left.\mathrm{P}(\mathrm{X} \geq 19)=8 \times 10^{-13}\right]$. The 43 overlapping contigs explain between 0 and $62.0 \%$ of the variance in methane production in the 16 independent validation animals $\left(\mathrm{R}^{2}\right.$ between contig count and residual methane production). The average amount of variance explained by the 43 contigs was $14.2 \%$. These variance estimates should be interpreted with caution, as the error variance in this analysis is large due to the small sample size (16). The contig that explains the most variance is still significant after a Bonferroni correction for multiple testing $(P=0.002)$.

To test if any taxa were over-represented in the contigs associated with methane production $(P<0.05$; negative binomial distribution), we used BLASTn and MEGAN4 to assign the methane-production associ- 
a)

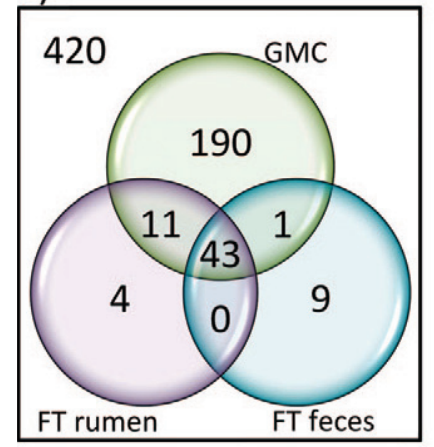

c)

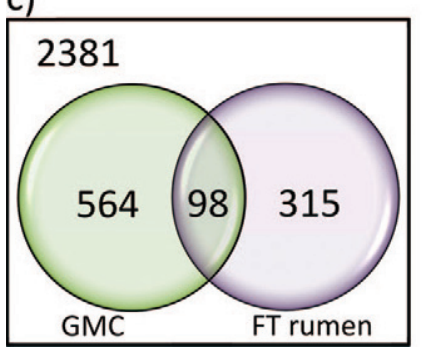

b)

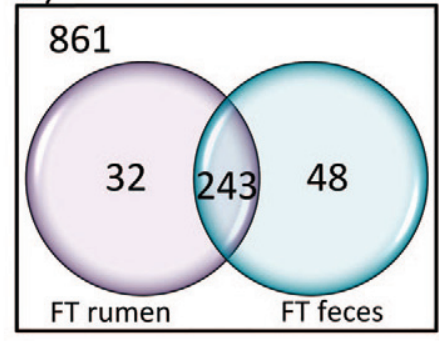

d)

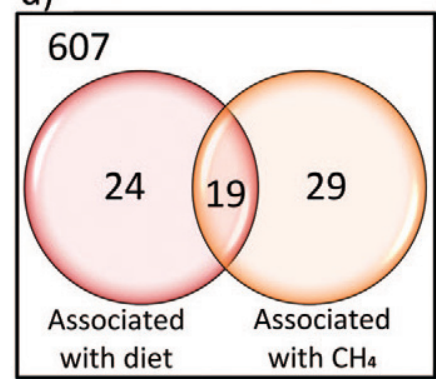

Figure 3. Contigs associated with diet or methane production. The number of significant contigs in each category is shown within the circle. The number in the overlaps between circles and contigs are significant in all or both experiments. The number outside the circles is the number of contigs not significant in any or either comparison. (a) Overlap between all 3 dietary comparisons (GMC, FT rumen, and FT feces; GMC = grapemarc; FT = fat and tannin). (b) Overlap between contigs that were significantly affected by diet in the FT experiment. The overlap is significantly more than expected by chance [cumulative hypergeometric test $\mathrm{P}(\mathrm{X}>243)=1.2 \times 10^{-125}$; that is, the probability $(\mathrm{P})$ that the overlap between the 2 lists $(\mathrm{X})$ is at least the observed number (243 in this case)]. (c) Overlap between contigs that were significantly affected by diet in the rumen comparisons. The overlap is significantly more than expected by chance [cumulative hypergeometric test $\left.P(X \geq 98)=8.6 \times 10^{-7}\right]$. (d) Contigs that are associated with the methane-mitigating diet in the GMC and FT studies (both rumen and feces) and residual methane $\left(\mathrm{CH}_{4}\right)$ production in a separate validation set of animals. The overlap is significantly more than expected by chance [cumulative hypergeometric test $\mathrm{P}(\mathrm{X} \geq 19)=8 \times 10^{-13}$ ]. Color version available in the online PDF.

ated contigs to taxa (Figure 4). We then compared the observed number in each taxa to what was expected by chance. Only Actinobacteria were significantly overrepresented in the contigs associated with methane production (Figure 5b).

\section{DISCUSSION}

Our results demonstrate that 2 different methanemitigating diets substantially altered the rumen microbiome profile, and that substantial overlap exists in the way these profiles were altered. We also found evidence that rumen microbial profiles from animals on these diets can be used to help identify microbiome biomarkers for low-methane-emitting cattle.

The relationships between samples were assessed using hierarchical clustering. The deep separation by diet in the rumen sample hierarchical clustering trees implies a strong effect of the diet on the rumen microbial profile, particularly in the FT experiment. This supports the results of the modeling analysis, in that it illustrates that the overall population of microbes was significantly altered by these diets. The lack of a consistent effect of the FT diet on the feces microbiome profiles may suggest that this diet did not affect the lower gastrointestinal tract microflora as dramatically as the rumen microflora. Alternatively, it may reflect the smaller amount of sequence which aligns to the database, reducing power to accurately cluster the samples. The hierarchical clustering assay is a broad comparison; a more sensitive assay may be able to detect alterations to the feces microbiome, as was observed by the modeling analysis.

The ability to predict the diet that the host animal was fed at the time of sampling (using metagenomic predictions) supports the hypothesis that these diets have substantially changed the rumen microbiome at a population-wide level and that these changes were similar for both methane-mitigating diets. The ability to predict across diets could be due to the common elements of the GMC and FT diets. The addition of fats to the diet has been shown to lower enteric methane production from ruminants (Blaxter and Czerkawski, 1966; Beauchemin et al., 2008; Martin et al., 2010; Moate et al., 2011), as has the addition of condensed tannins (Waghorn et al., 2002; Woodward et al., 2004); grapemarc contains both of these compounds (Fegeros and Kalaissakis, 1987). The fat and tannin components of grapemarc were reasons for its initial investigation as a methane-mitigating feed additive; hence, due to these components, a similar effect on the microbiota of the rumen may be expected.

Interestingly, our modeling results suggest that despite the extreme nature of the FT diet, either the animal itself or the initial microbial populations still have an effect on an individual's microbial profile. The animal effect on the rumen microbiome is approximately half of the animal effect that was calculated when repeated rumen samples were taken from the same cow at the same time in a previous experiment (Ross et al., 2012). Considering that the current study's repeat samples were altered by the methane-mitigating diet and the samples were also taken several weeks apart, the repeatability of sampling within animal is high. This finding suggests some stability of rumen microbial profiles may be seen over time, and is in concordance with the finding that the rumen ecosystems display substantial host specificity when almost the entire rumen contents was swapped between animals (Weimer 


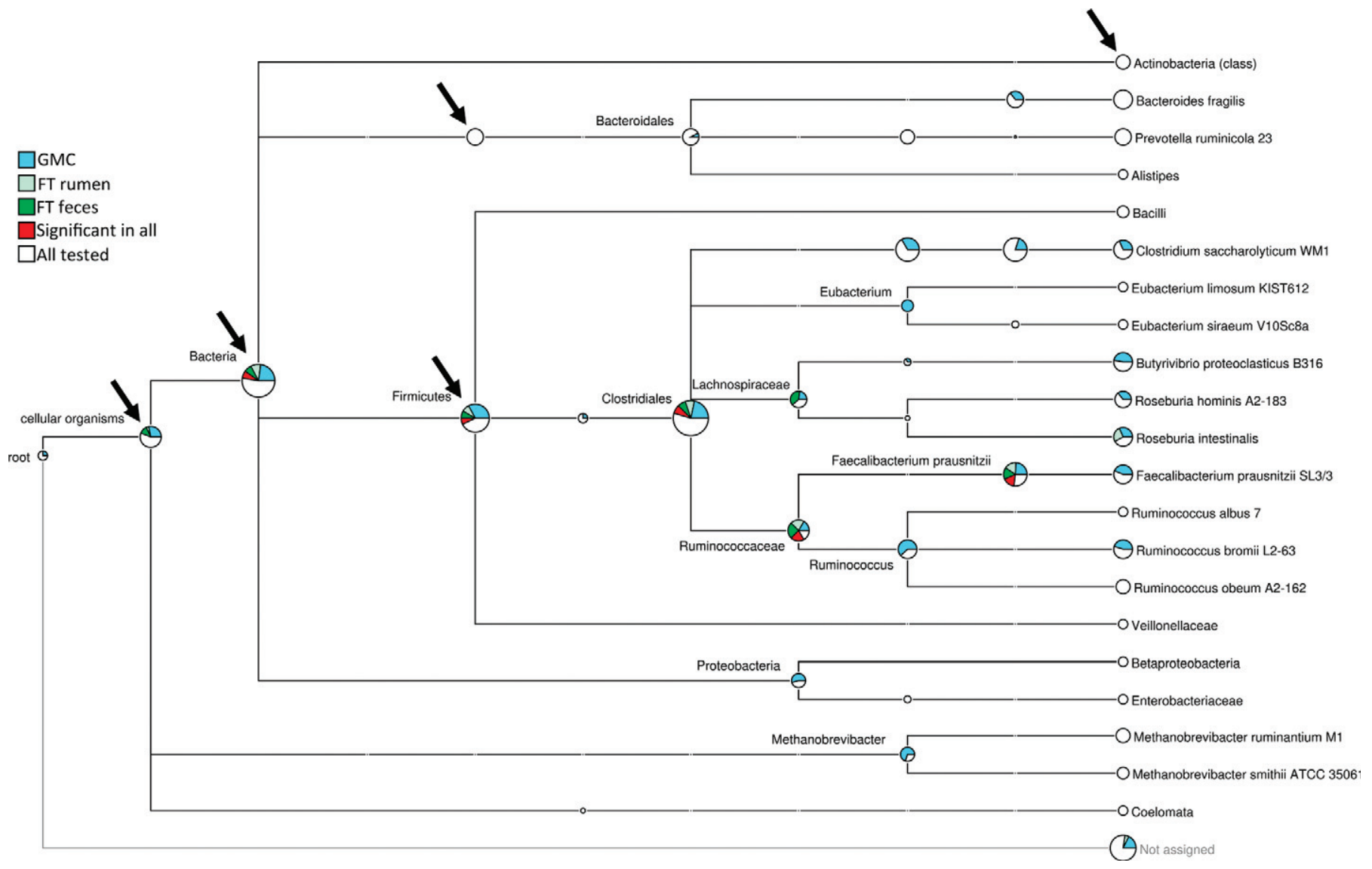

Figure 4. Significantly differentially represented taxa. Each contig was assigned to a taxon by using BLASTn (on an internal system) to the nucleotide database, then passing subsequent results to MEGAN4 (Huson et al., 2007; Huson et al., 2011; default settings). The taxa identified using contigs significantly associated with diet (in the GMC, FT rumen, and FT feces comparisons) are shown in red. The taxa identified using contigs that were significantly associated with residual methane production (general linear model, negative binomial distribution) are indicated with arrows. GMC = grapemarc; FT $=$ fat and tannin.

et al., 2010). Investigation of the temporal variation in the rumen microbiome warrants further exploration in a long-term study. Temporal stability in microbiome profiles is particularly important for the application of any useful rumen microbial biomarker that may be identified for a trait, such as enteric methane production.

One consideration for future work is that a larger rumen reference could be used for alignments. This is particularly important, as the majority of our sequence reads did not align to the database. This is likely because the contigs in the database we used represent approximately only $10 \%$ of the DNA sequence in the bovine metagenome of our test animals. Low realignment values to metagenomes are probably due to the structure of metagenome data, where the most abundant organisms receive deep sequence coverage and can, therefore, be assembled well. However, the large numbers of microorganisms that are present at low abundance are poorly assembled with incomplete sequence. Additionally, where the test animals and those used to assemble the contigs are independent, it may be reasonable to assume many microbial species are present in the test animals rumen that were not present in the animals used for assembly. The DNA extraction technique used will also alter which species are represented in the data (Carrigg et al., 2007). Other factors, such as breed, location, molecular techniques, and sample types (fiber-adherent microbes as compared with microbe in the fluid fraction of rumen digesta), may have influenced which species were represented in the data.

To ensure independence between the samples analyzed here and the reference data set, we limited our rumen database to sequence from animals not involved in either the FT or GMC study. Future analysis may include either the sequence analyzed here as part of the database, or sequence data from other studies as they becomes available. In addition to the data used here (Hess et al., 2011; Ross et al., 2012), other pos- 

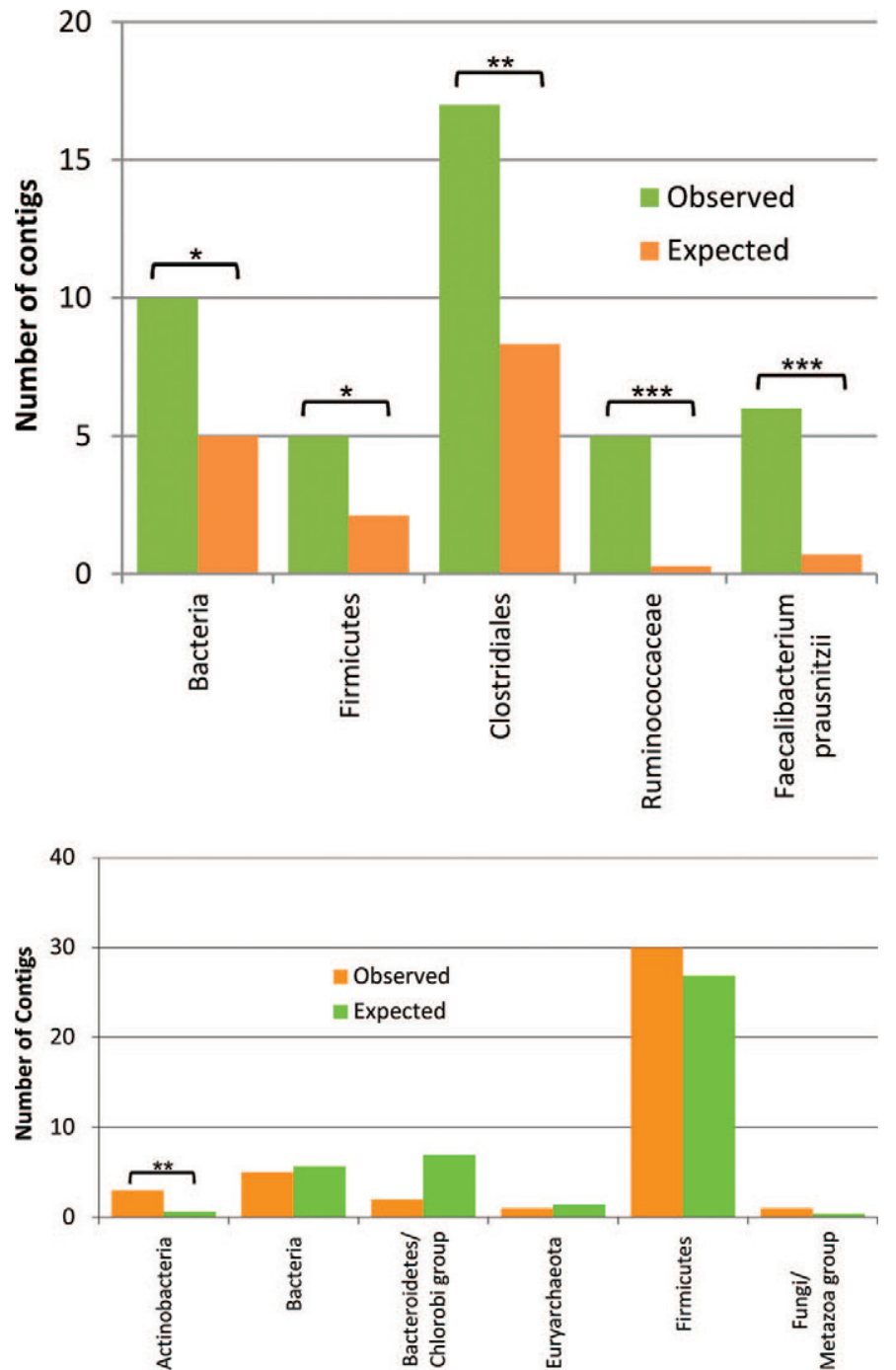

Figure 5. Taxa that are over represented in contigs associated with diet or methane production. Contigs were assigned to a taxa using BLASTn (on an internal system) to the nucleotide (nt) database. The output was assessed using MEGAN4 (Huson et al., 2007; Huson et al., 2011; default settings), before assignments were extracted. The expected numbers were calculated by assuming the same proportions as the 678 contigs tested. The observed value is the actual number of contigs MEGAN4 assigned to those taxa. Taxon values are not inclusive of contigs assigned to lower taxa. Significance was assessed using a chi square test, with one degree of freedom. ${ }^{*} P<0.05 ; * * P<0.005$ $* * * P<10^{-9}$. (A) Taxa identified in contig sets significantly associated with diet in all 3 dietary comparisons (GMC, FT rumen, and FT feces). (B) Taxa identified in contig sets significantly associated with residual methane production in the validation data set of 16 animals. $\mathrm{GMC}=$ grapemarc $; \mathrm{FT}=$ fat and tannin. Color version available in the online PDF.

sible sources of cattle metagenome data currently exist (Brulc et al., 2009; Durso et al., 2011; Li et al., 2011). A more complete database would have the advantage of using a greater proportion of the sequence for the analysis that is based on alignments to the rumen database. This would decrease the amount of data being discarded and, therefore, allow for the more efficient use of resources as the total sequencing depth required to obtain significant results would likely decrease.

Although only a basic functional analysis was conducted (KEGG pathways), a noticeable pattern was observed in the direction of changes as a result of the dietary additives. This finding is of interest because, until the advent of massively parallel sequencing, it was not possible to easily profile the functional characteristics of the rumen metagenome. Previously, studies of rumen microbial changes due to diet have been limited to phylogenetic marker genes, typically $16 \mathrm{~S}$ rRNA (a good example is Fernando et al., 2010). To gain functional insight, these studies assigned the $16 \mathrm{~S}$ sequence to a taxon and then inferred possible functional characteristics of the change from the genes known to occur in a related, culturable species. With untargeted (not 16S) massively parallel sequencing, we are now able to observe the functional changes directly.

The KEGG analysis demonstrated that the functional capacities of the microbiomes were altered in similar ways for both diets. Amino acid metabolism, carbohydrate metabolism, and translation were all significantly lower in the treatment animals on both diets. The observation that the 2 diets investigated here consistently altered the representation of the same KEGG pathways may be more informative then speculating on the relationship between individual pathways and methane production. This information on functional responses to methane-mitigating diets may aid research aimed at manipulation of the rumen microbiome to achieve lower greenhouse gas production. The similarities between diets are mirrored in the differential contig representation analysis, which found more overlap between diets than expected by chance.

In the contigs differentially represented in both the GMC and FT experiments, sequences similar to F. prausnitzii were overrepresented. Faecalibacterium prausnitzii (previously Fusobacterium prausnitzii; Duncan et al., 2002) is a common human gut microbe that has previously been implemented in studies of Crohn's disease (Jia et al., 2010; Sokol et al., 2008; Hansen et al., 2012) and, to a lesser extent, obesity (Balamurugan et al., 2010). However, F. prausnitzii has not been previously associated with methane production. The association between the methane-mitigating diets and F. prausnitzii-like bacteria warrants further investigation and validation, perhaps via more targeted methods such as quantitative PCR or sequencing of the $16 \mathrm{~S}$ genes present within the samples. Sequencing the 16S rRNA genes of the $F$. prausnitzii-like organism would assist in determining its degree of relatedness to $F$. prausnitzii strains present in public databases, such as nt.

The common reduction of the $F$. prausnitzii-like species and the pattern of similarities in the direction of 


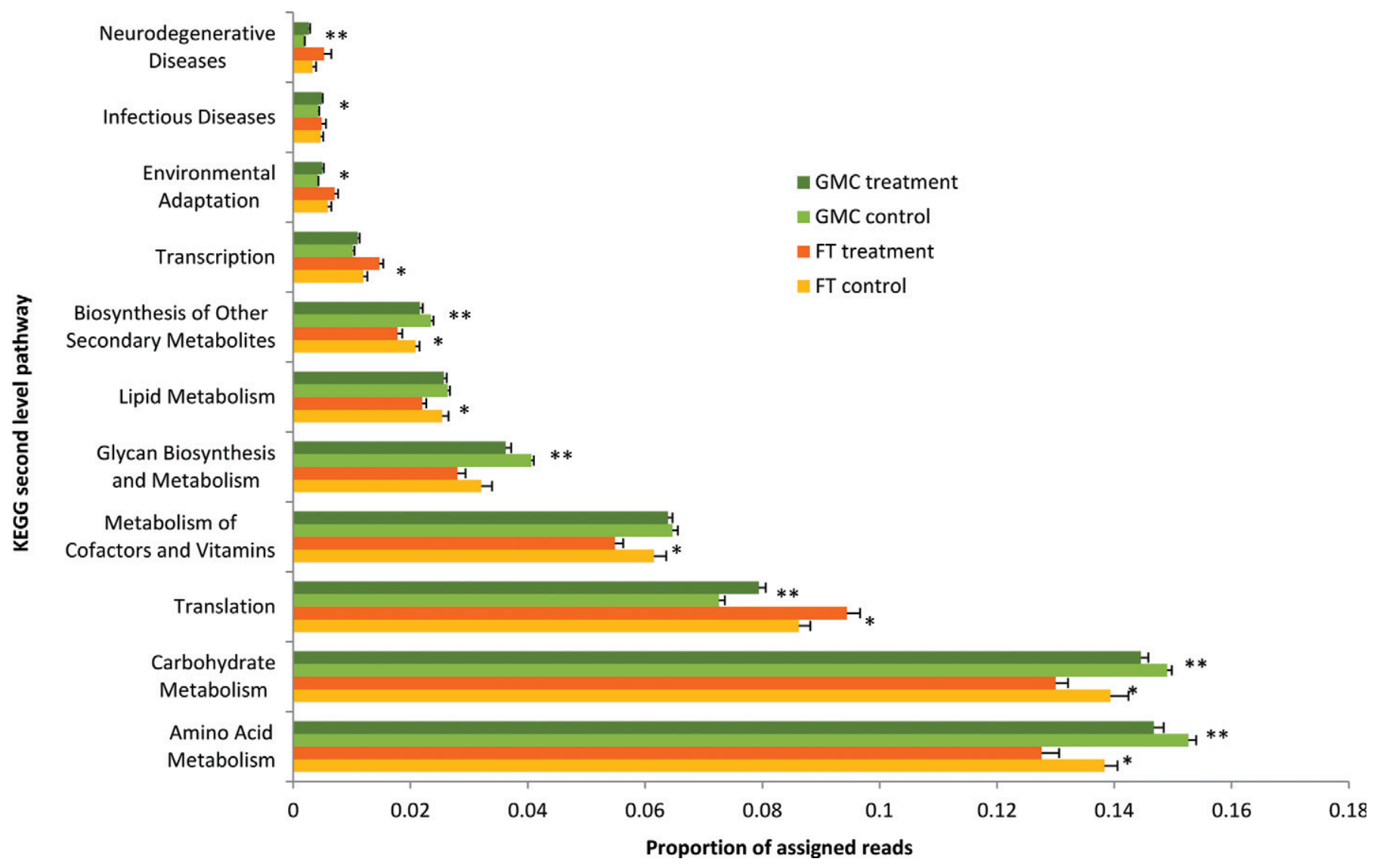

Figure 6. Comparison of KEGG (Kyoto Encyclopedia of Genes and Genomes; http://www.genome.jp/kegg/) second level pathways between treatments and controls. Relative proportion of pathways was determined by a BLASTx (on an internal system) to nr and then using MEGAN4 (Huson et al., 2007; Huson et al., 2011) to extract KEGG second level assignments. Error bars show SEM. Differences between control and treatment samples were tested for significance using a $t$-test. ${ }^{*} P<0.05 ;{ }^{*} P<0.01$. GMC $=$ grapemarc; FT $=$ fat and tannin. Color version available in the online PDF.

over-under representation of KEGG pathways suggest that the methane-mitigating diets have acted on the microbiome in similar ways, which concurs with the metagenomic prediction analysis results. These common microbiome alterations between the diets may be a result of common compounds (tannins and lipids) in the treatment diets that have introduced the same selective pressure on the rumen microbiome. An alternative explanation for the similarities between these diets is that they both altered the metagenomes to be more similar to naturally low-methane-emitting animals. These 2 explanations are not mutually exclusive and a combination of both is possible.

If the treatments have altered the microbiomes to resemble low-methane-emitting profiles then it should be possible to use the differentially represented contigs to identify biomarkers for low methane production. We found that the contigs that were differentially represented between diets showed significant enrichment for associations with methane production level in an inde- pendent set of animals. This identification of methaneassociated contigs illustrates how methane-mitigating diets can be used to identify biomarkers for low methane production. This finding, and the ability to use these samples as a reference for predicting methane in the 16 independent animals (Ross et al., 2013), illustrates the value in examining changes in the rumen microbiome associated with reducing enteric methane production via dietary additives. These additives (which may be a tool for reducing methane production) are able to aid in the identification of naturally occurring biomarkers for low methane production. Ideally, these contigs will be validated in a large data set, as the limited number of animals used here limits the power of this study to detect subtle associations.

As most methane is produced in the rumen, a biomarker for low methane production is most likely to be associated with the rumen environment or microbiome. However, the difficulty in sampling rumen contents from large numbers of animals means that other sample 
types should also be investigated. Any biomarker for low methane emissions, or methane yield, or any other trait of interest, must be easily obtainable. Therefore, despite the differences between the rumen and fecal microbiomes (Ross et al., 2012), analysis of the fecal microbiome was conducted in this study to further our understanding of the relationship between the rumen and fecal microbial populations and how they relate to methane production. The overlap in contigs associated with the methane mitigating diets between the rumen fluid and feces, and the subsequent association of those contigs with residual methane production in the independent cohort of animals suggests that it may be possible to identify methane production biomarkers in the fecal microbiome. These findings suggest it would be valuable to test rumen biomarkers on the fecal metagenome, particularly considering the increased ease of sampling that fecal biomarkers present. The physiological cause for the fecal biomarkers' association with methane production is unknown, but it could be speculated that either the biomarkers are traveling through the animal's digestive tract from the rumen, or that individual animals exert a common selective pressure on both the rumen and lower gastrointestinal tract environment.

\section{CONCLUSIONS}

This study examined changes in the bovine rumen and fecal metagenomes of 2 methane-mitigating diets. Significant changes in the rumen microbiome profiles were detected via a modeling approach and by hierarchical clustering. A general similarity in the way that the microbiomes were altered was observed, as illustrated by the ability to predict the diet of the host animal across experiment and the significant overlap in differentially represented contigs between the 2 experiments. The same patterns of microbiome alterations were also detected in the fecal samples of one experiment. Finally, we were able to provide supportive evidence, through validation in an independent set of animals, that these diets can be used to identify contigs associated with natural variation in enteric methane production of cattle. The analysis of the microbiome changes in the GMC and FT experiments has provided this study with greater power to detect patterns of microbial population change that are associated with lower methane production than could be achieved by interrogating only 1 methane-mitigating feed additive. This added power has allowed the identification of contigs that warrant further investigation as biomarkers for low-methane-emitting cattle.

\section{ACKNOWLEDGMENTS}

The authors thank Josquin Tibbits (Department of Environment and Primary Industries, Victoria, Australia) and Steve Petrovski (Peter MacCallum Cancer Centre, formally Department of Environment and Primary Industries Victoria) for laboratory assistance. Thank you also to Charlotte Anderson, Sami Hakim, and the staff of Department of Environment and Primary Industries, Ellinbank, that were involved in sampling. Funding for this work was provided by the Dairy Futures Cooperative Research Centre and The Geoffrey Gardiner Dairy Foundation (Melbourne, Victoria, Australia). Funding for the two parent experiments was provided by Meat and Livestock Australia (Sydney, NSW, Australia), the Victorian Department of Environment and Primary Industries, and the Australian Government Department of Agriculture, Fisheries and Forestry (Canberra, Australia) under its Australia's Future Climate Change research program.

\section{REFERENCES}

Attwood, G., and C. McSweeney. 2008. Methanogen genomics to discover targets for methane mitigation technologies and options for alternative $\mathrm{H}_{2}$ utilisation in the rumen. Aust. J. Exp. Agric. 48:28-37.

Balamurugan, R., G. George, J. Kabeerdoss, J. Hepsiba, A. M. Chandragunasekaran, and B. S. Ramakrishna. 2010. Quantitative differences in intestinal Faecalibacterium prausnitzii in obese Indian children. Br. J. Nutr. 103:335-338.

Beauchemin, K., M. Kreuzer, F. O'Mara, and T. McAllister. 2008 Nutritional management for enteric methane abatement: A review. Aust. J. Exp. Agric. 48:21-27.

Bhatt, V. D., S. S. Dande, N. V. Patil, and C. G. Joshi. 2013. Molecular analysis of the bacterial microbiome in the forestomach fluid from the dromedary camel (Camelus dromedarius). Mol. Biol. Rep. 40:3363-3371.

Blaxter, K. L., and J. Czerkawski. 1966. Modification of the methane production of the sheep by supplementation of its diet. J. Sci. Food Agric. 17:417-421.

Brulc, J. M., D. A. Antonopoulos, M. E. Berg Miller, M. K. Wilson, A. C. Yannarell, E. A. Dinsdale, R. E. Edwards, E. D. Frank, J. B. Emerson, P. Wacklin, P. M. Coutinho, B. Henrissat, K. E. Nelson, and B. A. White. 2009. Gene-centric metagenomics of the fiber-adherent bovine rumen microbiome reveals forage specific glycoside hydrolases. Proc. Natl. Acad. Sci. USA 106:1948-1953.

Carrigg, C., O. Rice, S. Kavanagh, G. Collins, and V. O'Flaherty. 2007. DNA extraction method affects microbial community profiles from soils and sediment. Appl. Microbiol. Biotechnol. 77:955-964.

Dai, X., Y. Zhu, Y. Luo, L. Song, D. Liu, L. Liu, F. Chen, M. Wang, J. Li, X. Zeng, Z. Dong, S. Hu, L. Li, J. Xu, L. Huang, and X. Dong. 2012. Metagenomic insights into the fibrolytic microbiome in yak rumen. PLoS ONE 7:e40430.

de Menezes, A. B., E. Lewis, M. O'Donovan, B. F. O'Neill, N. Clipson, and E. M. Doyle. 2011. Microbiome analysis of dairy cows fed pasture or total mixed ration diets. FEMS Microbiol. Ecol. $78: 256-265$.

Dehority, B. 2003. Rumen Microbiology. Nottingham University Press, Nottingham, UK.

Duncan, S. H., G. L. Hold, H. J. M. Harmsen, C. S. Stewart, and H. J. Flint. 2002. Growth requirements and fermentation products of Fusobacterium prausnitzii, and a proposal to reclassify it as Fae- 
calibacterium prausnitzii gen. nov., comb. nov. Int. J. Syst. Evol. Microbiol. 52:2141-2146.

Durso, L. M., G. P. Harhay, J. L. Bono, and T. P. L. Smith. 2011. Virulence-associated and antibiotic resistance genes of microbial populations in cattle feces analyzed using a metagenomic approach. J. Microbiol. Methods 84:278-282.

Engelbrektson, A., V. Kunin, K. C. Wrighton, N. Zvenigorodsky, F. Chen, H. Ochman, and P. Hugenholtz. 2010. Experimental factors affecting PCR-based estimates of microbial species richness and evenness. ISME J. 4:642-647.

Fegeros, K., and P. Kalaissakis. 1987. The nutritive value of grape marc for sheep. World Rev. Anim. Prod. 23:17-22.

Fernando, S. C., H. T. Purvis II, F. Z. Najar, L. O. Sukharnikov, C. R. Krehbiel, T. G. Nagaraja, B. A. Roe, and U. DeSilva. 2010. Rumen microbial population dynamics during adaptation to a highgrain diet. Appl. Environ. Microbiol. 76:7482-7490.

Frey, J. C., A. N. Pell, R. Berthiaume, H. Lapierre, S. Lee, J. K. Ha, J. E. Mendell, and E. R. Angert. 2010. Comparative studies of microbial populations in the rumen, duodenum, ileum and faeces of lactating dairy cows. J. Appl. Microbiol. 108:1982-1993.

Gilmour, A. R., B. J. Gogel, B. R. Cullis, and R. Thompson. 2006. ASReml User Guide Release 2.0. VSN International Ltd., Hemel Hempstead, UK.

Grainger, C., T. Clarke, S. M. McGinn, M. J. Auldist, K. A. Beauchemin, M. C. Hannah, G. C. Waghorn, H. Clark, and R. J. Eckard. 2007. Methane emissions from dairy cows measured using the sulfur hexafluoride $\left(\mathrm{SF}_{6}\right)$ tracer and chamber techniques. J. Dairy Sci. 90:2755-2766.

Hansen, R., R. K. Russell, C. Reiff, P. Louis, F. McIntosh, S. H. Berry, I. Mukhopadhya, W. M. Bisset, A. R. Barclay, J. Bishop, D. M. Flynn, P. McGrogan, S. Loganathan, G. Mahdi, H. J. Flint, E. M. El-Omar, and G. L. Hold. 2012. Microbiota of de-novo pediatric IBD: Increased Faecalibacterium prausnitzii and reduced bacterial diversity in Crohn's but not in ulcerative colitis. Am. J. Gastroenterol. 107:1913-1922.

Hegarty, R. S., J. P. Goopy, R. M. Herd, and B. McCorkell. 2007. Cattle selected for lower residual feed intake have reduced daily methane production. J. Anim. Sci. 85:1479-1486.

Henderson, C. R. 1984. Applications of Linear Models in Animal Breeding. University of Guelph Press, Guelph, Canada.

Hernandez-Sanabria, E., L. A. Goonewardene, Z. Wang, O. N. Durunna, S. S. Moore, and L. L. Guan. 2012. Impact of feed efficiency and diet on adaptive variations in the bacterial community in the rumen fluid of cattle. Appl. Environ. Microbiol. 78:1203-1214.

Hess, M., A. Sczyrba, R. Egan, T.-W. Kim, H. Chokhawala, G. Schroth, S. Luo, D. S. Clark, F. Chen, T. Zhang, R. I. Mackie, L. A. Pennacchio, S. G. Tringe, A. Visel, T. Woyke, Z. Wang, and E. M. Rubin. 2011. Metagenomic discovery of biomass-degrading genes and genomes from cow rumen. Science 331:463-467.

Hong, S., J. Bunge, C. Leslin, S. Jeon, and S. S. Epstein. 2009. Polymerase chain reaction primers miss half of rRNA microbial diversity. ISME J. 3:1365-1373.

Huber, J. A., H. G. Morrison, S. M. Huse, P. R. Neal, M. L. Sogin, and D. B. Mark Welch. 2009. Effect of PCR amplicon size on assessments of clone library microbial diversity and community structure. Environ. Microbiol. 11:1292-1302.

Huson, D. H., A. F. Auch, J. Qi, and S. C. Schuster. 2007. MEGAN analysis of metagenomic data. Genome Res. 17:377-386.

Huson, D. H., S. Mitra, H.-J. Ruscheweyh, N. Weber, and S. C. Schuster. 2011. Integrative analysis of environmental sequences using MEGAN4. Genome Res. 21:1552-1560.

IPCC. 2007. Agriculture. Contribution of working group III to the fourth assessment report of the Intergovernmental Panel on Climate Change. Chapter 8 in IPCC Fourth Assessment Report. B. Metz, O. R. Davidson, P. R. Bosch, R. Dave, and L. A. Meyer, ed. Cambridge University Press, Cambridge, UK.

Jia, W., R. N. Whitehead, L. Griffiths, C. Dawson, R. H. Waring, D. B. Ramsden, J. O. Hunter, and J. A. Cole. 2010. Is the abundance of Faecalibacterium prausnitzii relevant to Crohn's disease? FEMS Microbiol. Lett. 310:138-144.
Lassey, K., F. Ulyatt, R. Martin, C. Walker, and I. Shelton. 1997. Methane emissions measured directly from grazing livestock in New Zealand. Atmos. Environ. 31:2905-2914.

Li, H., and R. Durbin. 2009. Fast and accurate short read alignment with Burrows-Wheeler transform. Bioinformatics 25:1754-1760.

Li, R. W., S. Wu, W. Li, Y. Huang, and L. C. Gasbarre. 2011. Metagenome plasticity of the bovine abomasal microbiota in immune animals in response to Ostertagia ostertagi infection. PLoS ONE $6: \mathrm{e} 24417$.

Martin, C., D. P. Morgavi, and M. Doreau. 2010. Methane mitigation in ruminants: From microbe to the farm scale. Animal 4:351-365.

Moate, P. J., S. R. O. Williams, C. Grainger, M. C. Hannah, E. N. Ponnampalam, and R. J. Eckard. 2011. Influence of cold-pressed canola, brewers grains and hominy meal as dietary supplements suitable for reducing enteric methane emissions from lactating dairy cows. Anim. Feed Sci. Technol. 166-167:254-264.

Moate, P. J., S. R. O. Williams, B. E. Ribaux, and W. J. Wales. 2012. Feeding grape marc to dairy cows suppresses methane emissions. Pages 454-455 in Proc. 5th Australas. Dairy Sci. Symp. http:// www.adssymposium.com.au/inewsfiles/ADSS_Final_Proceedings. pdf.

Murray, R. M., A. M. Bryant, and R. A. Leng. 1976. Rates of production of methane in the rumen and large intestine of sheep. Br. J. Nutr. 36:1-14.

Pitta, D. W., W. Pinchak, S. Dowd, J. Osterstock, V. Gontcharova, E. Youn, K. Dorton, I. Yoon, B. Min, J. D. Fulford, T. Wickersham, and D. Malinowski. 2010. Rumen bacterial diversity dynamics associated with changing from bermudagrass hay to grazed winter wheat diets. Microb. Ecol. 59:511-522.

Pope, P. B., A. K. Mackenzie, I. Gregor, W. Smith, M. A. Sundset, A. C. McHardy, M. Morrison, and V. G. Eijsink. 2012. Metagenomics of the Svalbard reindeer rumen microbiome reveals abundance of polysaccharide utilization loci. PLoS ONE 7:e38571.

R Development Core Team. 2011. R: A Language and Environment for Statistical Computing. R Foundation for Statistical Computing, Vienna, Austria.

Robinson, M. D., D. J. McCarthy, and G. K. Smyth. 2010. edgeR: A Bioconductor package for differential expression analysis of digital gene expression data. Bioinformatics 26:139-140.

Robinson, M. D., and G. K. Smyth. 2007. Moderated statistical tests for assessing differences in tag abundance. Bioinformatics 23:2881-2887.

Robinson, M. D., and G. K. Smyth. 2008. Small-sample estimation of negative binomial dispersion, with applications to SAGE data. Biostatistics 9:321-332.

Ross, E. M., P. J. Moate, C. R. Bath, S. E. Davidson, T. I. Sawbridge, K. M. Guthridge, B. G. Cocks, and B. J. Hayes. 2012. High throughput whole rumen metagenome profiling using untargeted massively parallel sequencing. BMC Genet. 13:53.

Ross, E. M., P. J. Moate, L. Marett, B. G. Cocks, and B. J. Hayes. 2013. Rumen microbiome profiles predict enteric methane production from dairy cattle. Page 312 in Proc. 5th Greenhouse Gases and Animal Agriculture Conference. Cambridge University Press, Dublin, Ireland.

Singh, K. M., V. B. Ahir, A. K. Tripathi, U. V. Ramani, M. Sajnani, P. G. Koringa, S. Jakhesara, P. R. Pandya, D. N. Rank, D. S. Murty, R. K. Kothari, and C. G. Joshi. 2012. Metagenomic analysis of Surti buffalo (Bubalus bubalis) rumen: A preliminary study. Mol. Biol. Rep. 39:4841-4848.

Sokol, H., B. Pigneur, L. Watterlot, O. Lakhdari, L. G. BermudezHumaran, J. J. Gratadoux, S. Blugeon, C. Bridonneau, J. P. Furet, G. Corthier, C. Grangette, N. Vasquez, P. Pochart, G. Trugnan, G. Thomas, H. M. Blottiere, J. Dore, P. Marteau, P. Seksik, and P. Langella. 2008. Faecalibacterium prausnitzii is an anti-inflammatory commensal bacterium identified by gut microbiota analysis of Crohn disease patients. Proc. Natl. Acad. Sci. USA 105:1673116736 .

Stiverson, J., M. Morrison, and Z. Yu. 2011. Populations of select cultured and uncultured bacteria in the rumen of sheep and the effect of diets and ruminal fractions. Int. J. Microbiol. 2011:750613. 
Sun, W. 2012. A statistical framework for eQTL mapping using RNAseq data. Biometrics 68:1-11.

Venables, W. N., and B. D. Ripley. 2002. Modern Applied Statistics with S. 4th ed. Springer, New York, NY.

Waghorn, G., M. Tavendale, and D. Woodfield. 2002. Methanogenesis from forages fed to sheep. Pages 167-171 in Proc. 64th N. Z. Grassl. Assoc. http://www.grassland.org.nz/publications/ nzgrassland_publication_479.pdf.

Weimer, P. J., D. Stevenson, H. Mantovani, and S. Man. 2010. Host specificity of the ruminal bacterial community in the dairy cow following near-total exchange of ruminal contents. J. Dairy Sci. 93:5902-5912

Whitford, M. F., R. J. Forster, C. E. Beard, J. Gong, and R. M. Teather. 1998. Phylogenetic analysis of rumen bacteria by comparative sequence analysis of cloned $16 \mathrm{~S}$ rRNA genes $ß$. Anaerobe 4:153-163.

Woodward, S., G. Waghorn, and P. Laboyrie. 2004. Condensed tannins in birdsfoot trefoil (Lotus corniculatus) reduced methane emissions from dairy cows. Proc. N.Z. Soc. Anim. Prod. 64:160-164. 